\title{
Intervenção Psicológica em Pacientes Submetidos a Angioplastia Coronária: Ensaio Randomizado
}

\author{
Rosângela Soares ${ }^{1}$, George Cesar Ximenes Meireles², Luciano Maurício de Abreu Filho', \\ Antônio Artur da Cruz Forte', Marcos Kiyoshi Sumita', Elaine Ortiz de Moraes ${ }^{3}$
}

\section{RESUMO}

Introdução: Elevado grau de estresse emocional é usualmente encontrado no atendimento de pacientes que aguardam a realização de angioplastia coronária. Nosso objetivo principal foi avaliar a eficácia do atendimento psicológico na redução do estresse emocional em pacientes submetidos a angioplastia coronária. Método: Trata-se de estudo prospectivo, sequencial, randomizado. A casuística da presente análise foi composta de 128 pacientes portadores de insuficiência coronária aguda ou crônica, divididos em dois grupos: grupo A, 66 pacientes que receberam atendimento psicológico hospitalar pré-intervenção, e grupo B, 62 pacientes que realizaram angioplastia coronária sem atendimento psicológico hospitalar. Os dois grupos responderam a um inventário específico para avaliar se havia presença ou não de estresse emocional antes e após a angioplastia. Resultados: Na repetição do inventário, observou-se que no grupo A $74 \%$ dos pacientes não apresentavam estresse emocional e $26 \%$ continuavam com estresse. No grupo B, na repetição do inventário, observou-se que $6 \%$ dos pacientes não apresentavam estresse emocional e $94 \%$ continuavam com estresse. Conclusão: $O$ atendimento psicológico em pacientes submetidos a angioplastia coronária foi eficaz na redução do estresse.

DESCRITORES: Angioplastia. Estresse psicológico. Psicologia. Humanização da assistência.

1 Serviço de Cardiologia Intervencionista - Hospital Stella Maris Guarulhos, SP, Brasil.

2 Serviço de Cardiologia Intervencionista - Hospital do Servidor Público Estadual - São Paulo, SP, Brasil.

${ }^{3}$ Instituto de Assistência Médica ao Servidor Público Estadual (lamspe) - São Paulo, SP, Brasil.

Correspondência: Rosangela Soares. Rua Conselheiro Saraiva, 812 Santana - São Paulo, SP, Brasil - CEP 02037-021

E-mail: ropsique@ig.com.br

Recebido em: 10/6/2010 • Aceito em: 27/8/2010

Trabalho realizado como parte dos requisitos para obtenção do título de Mestre em Ciências da Saúde pelo Instituto de Assistência ao Servidor Público Estadual (lamspe), cuja defesa/aprovação ocorreu em 21 de junho de 2010. Suporte financeiro: Coordenação de Aperfeiçoamento de Pessoal de Nível Superior (CAPES).

\section{ABSTRACT}

\section{Psychological Intervention in Patients Undergoing} Coronary Angioplasty: A Randomized Trial

Background: High rates of emotional stress are usually observed in the care of patients waiting for coronary angioplasty. Our main objective was to evaluate the effectiveness of psychological treatment in reducing emotional stress in patients undergoing coronary angioplasty. Method: This is a prospective, sequential, randomized study. This study included 128 patients with acute or chronic coronary artery disease who were divided into two groups: group A included 66 patients who received psychological treatment prior to coronary angioplasty, and group B with 62 patients who underwent coronary angioplasty without psychological treatment. Both groups answered a specific questionnaire to assess whether there was emotional stress prior and after angioplasty. Results: When the questionnaire was repeated, it was observed that in group A, $74 \%$ of the patients did not have emotional stress, whereas it was present in $26 \%$ of the patients. In group B, when the questionnaire was repeated, $6 \%$ of the patients did not have emotional stress, whereas it was present in $94 \%$ of them. Conclusion: Psychological treatment in patients undergoing coronary angioplasty was effective in reducing stress.

KEY-WORDS: Angioplasty. Stress, psychological. Psychology. Humanization of assistance.
0 estresse é uma atitude biológica necessária para a adaptação de um organismo. Fisicamente o estresse aparece quando o organismo é submetido a nova situação, como uma cirurgia, ou, do ponto de vista psicoemocional, quando há uma situação percebida como ameaça, pela qual ele terá que lutar e se adaptar. Portanto, o estresse emocional é um mecanismo indispensável para a adaptação à vida. $^{1}$

As fases do estresse emocional iniciam com a fase do alerta, considerada a fase positiva do estresse, em que o indivíduo se energiza por meio da produção de adrenalina, a sobrevivência é preservada e uma sensação de plenitude frequentemente é alcançada. $\mathrm{Na}$ segunda fase, a da resistência, o indivíduo automatica- 
mente tenta lidar com seus estressores de modo a manter sua homeostase interna. Se os fatores estressantes persistirem em frequência ou intensidade, há uma quebra na resistência do indivíduo, quando se inicia a terceira fase, a da quase-exaustão. Nessa fase, o processo do adoecimento se inicia e os órgãos que possuírem maior vulnerabilidade genética ou adquirida passam a mostrar sinais de deterioração. Se não há alívio para o estresse por meio da remoção dos estressores ou pelo uso de estratégias de enfrentamento, o estresse atinge sua fase final, a da exaustão, quando doenças graves podem ocorrer nos órgãos mais vulneráveis, como infarto, úlceras, psoríase, depressão e outros. $^{2}$

Existem evidências convincentes de que fatores psicossociais, tais como ansiedade, isolamento social, estresse e personalidade tipo A, contribuem significativamente para a patogênese da aterosclerose e eventos cardiovasculares. Esses fatores psicossociais podem ativar mecanismos biológicos de estresse crônico, aumentando a atividade do eixo hipotálamo-hipófise-adrenal, do sistema simpático e da inflamação, os quais causam aumento da coagulação sanguínea e têm efeito aterogênico. ${ }^{3-6}$

Estudos recentes demonstraram que a ansiedade é um preditor independente para doença arterial coronária. ${ }^{7,8}$ Roest et al. ${ }^{7}$ demonstraram, após meta-análise da associação entre ansiedade e incidência de doença arterial coronária em pessoas inicialmente sadias, que pessoas ansiosas têm probabilidade $25 \%$ e $50 \%$ maior de risco de doença arterial coronária [hazard ratio (HR) 1,26; $\mathrm{P}<0,0001]$ e de morte cardíaca (HR 1,48; $\mathrm{P}=0,003)$, respectivamente. Janszky et al. ${ }^{8}$ observaram, em seguimento por 37 anos de 50 mil homens examinados para o serviço militar na Suécia, que a depressão não é preditor de doença arterial coronária (HR 1,03; $\mathrm{P}=\mathrm{NS}$ ) ou de infarto agudo do miocárdio (HR 1,03; $\mathrm{P}=\mathrm{NS}$ ), mas que a ansiedade proporciona duas vezes mais chance de apresentar doença arterial coronária ou infarto agudo do miocárdio (HR 2,17 e 2,51, respectivamente; $\mathrm{P}<0,001)$.

A carga emocional de um paciente quando se estabelece uma doença crônica é equivalente à de grande perda, que pode ser relacionada ao luto. Existe grande insegurança quanto ao futuro e quanto à volta ao trabalho, já que muitos estão em plena atividade profissional. São questões angustiantes que refletem o medo dos danos causados pela doença cardíaca e a forma como esses danos vão interferir na qualidade de suas vidas. ${ }^{9}$

Nessas situações, quando o paciente é informado sobre o diagnóstico de doença coronária e sobre a necessidade de se submeter a angioplastia, sua reação emocional pode interferir na evolução da doença, na adesão ao tratamento e na mudança de hábito de vida. Isso porque cada paciente apresenta suas próprias fantasias e mecanismos de defesa como forma de en- frentar a doença, vivenciando esse fato de maneira única e individual. ${ }^{9,10}$

Da mesma forma, estar dentro de um hospital, internado, ou em ambulatório, buscando o diagnóstico, e saber-se portador de uma doença rememora vivências passadas, sinaliza a realidade da morte, inicia a ideia da incapacidade do cuidar de si mesmo, conduz à revisão de valores de vida, o que, com frequência, traz dor e amedronta. Esse medo é ainda maior quando o órgão a ser tratado é o coração. Isso porque o coração é repleto de significados e simbologias que envolvem emoções pessoais, culturais e psicossociais. Assim, o paciente que se depara com uma intervenção cardíaca necessariamente tem que enfrentar os aspectos objetivos e simbólicos de sua vida. ${ }^{11}$

Alguns autores sugerem que a sedação durante a angioplastia coronária é uma maneira de tornar o procedimento mais aceitável pelo paciente, conforme demonstrado em estudo comparativo da realização de angioplastia coronária com e sem sedação, observando que no grupo com sedação a angioplastia se caracterizou por experiência indolor e segura. ${ }^{12}$

O atendimento psicológico hospitalar direcionado aos pacientes que vão se submeter a angioplastia coronária tem como base a psicoterapia breve de apoio, um tipo de ação terapêutica que visa a diminuir a ansiedade do paciente. Durante os atendimentos busca-se entender o que o paciente sabe a respeito de sua doença e sobre o procedimento da angioplastia coronária, com o objetivo de esclarecer dúvidas e questões gerais pertinentes ao procedimento. Quando existem dúvidas específicas às questões orgânicas, estimula-se que as mesmas sejam solucionadas com a equipe médica. Procura-se também despertar no paciente a importância da prevenção dos fatores de risco da doença arterial coronária e esclarecer como o paciente pode participar ativamente na busca de melhor qualidade de vida. Por fim, busca-se dar suporte às angústias e medos do paciente diante da hospitalização e suas decorrências. ${ }^{13,14}$

O objetivo do presente estudo foi avaliar a eficácia do atendimento psicológico hospitalar na redução do estresse emocional em pacientes submetidos a angioplastia coronária.

\section{MÉTODO}

Trata-se de um estudo prospectivo sequencial, randomizado com pacientes provenientes do Serviço de Hemodinâmica e Cardiologia Intervencionista do Hospital Stella Maris, Guarulhos, SP. Na enfermaria dessa instituição foram realizados os atendimentos psicológicos hospitalares e aplicou-se o Inventário de Sintomas de Stress para Adultos (ISSL)², e no laboratório de hemodinâmica foram realizadas as intervenções coronárias percutâneas. O protocolo de estudo, aprovado pelo Comitê de Ética em Pesquisa do Instituto de 
Assistência Médica ao Servidor Público Estadual, foi conduzido de acordo com os princípios da Declaração de Helsinque. O termo de consentimento livre e esclarecido foi obtido de todos os pacientes.

A casuística foi composta de 128 pacientes portadores de síndrome coronária aguda ou angina estável, elegíveis para revascularização miocárdica percutânea. Os pacientes encontravam-se internados na enfermaria para serem submetidos a intervenção coronária percutânea, procedimento indicado por cardiologistas clínicos e intervencionistas. Foram excluídos os pacientes que, anteriormente ao estudo, já haviam recebido atendimento psicológico hospitalar pela pesquisadora responsável.

A randomização foi realizada pela obtenção de uma sequência aleatória de números, em duas colunas, obtidos em endereço eletrônico especializado. Seguindo a sequência da randomização, os pacientes foram alocados para os grupos com (grupo A) e sem (grupo B) atendimento psicológico.

Aplicou-se o inventário ISSL, constituído por dados para identificação do paciente e 53 questões que visam a identificar de modo objetivo os sintomas que o paciente apresenta. Esse inventário avalia, também, se o paciente possui sintomas de estresse, e, em caso positivo, distingue se o sintoma é somático ou psicológico e a fase em que se encontra (nas últimas 24 horas, na última semana ou no último mês). Os sintomas listados são típicos de cada fase do estresse emocional (alerta, resistência, quase exaustão e exaustão), sendo 37 itens de natureza somática e 19, psicológica.*

Na fase pré-angioplastia, foram explicados aos pacientes os motivos da pesquisa, e para os que concordaram em fazer parte do estudo aplicou-se o termo de consentimento livre e esclarecido. Em seguida, esses pacientes foram randomizados, sendo aplicado o inventário ISSL. Os pacientes do grupo A receberam atendimento psicológico, que consistia de uma única sessão em que as dúvidas e questões gerais eram respondidas, sendo oferecido suporte às angústias e aos medos antes do encaminhamento à angioplastia coronária. Os pacientes do grupo B eram encaminhados diretamente à intervenção.

Na fase pós-angioplastia, um mês após a intervenção, os dois grupos responderam ao mesmo inventário ISSL. Para o grupo A, foi adicionada a questão: "O atendimento psicológico antes da angioplastia foi: Eficaz ( ) Não eficaz ( )".

* É proibida a divulgação do conteúdo de testes psicológicos e sua utilização é restrita ao profissional de psicologia, de acordo com o Decreto no 53.464 de 21/1/1964, que regulamenta a Lei Federal no 4.119 , a qual dispõe sobre a profissão de psicólogo.
O cálculo da amostra foi obtido pelo programa Primer, com estimativa de redução do estresse emocional de $25 \%$. Por meio desse cálculo foi obtida uma amostra de 57 pacientes para cada grupo. Como medida preventiva, considerando-se a possibilidade de eventuais perdas nos seguimentos em 30 dias após a angioplastia coronária, foram acrescentados, aproximadamente, $10 \%$ de pacientes em cada grupo. As variáveis quantitativas apresentam-se como médias e desvios padrão e as variáveis qualitativas, como frequências absolutas e relativas. Para testar a hipótese de igualdade nos dois grupos nos momentos pré e pós-angioplastia, utilizou-se o teste de qui-quadrado, com correção de Yates, e para comparação entre a primeira e a segunda fases utilizou-se o teste de McNemar. O nível de significância adotado foi de $5 \%(P<0,05)$.

\section{RESULTADOS}

No período da coleta de dados, entre março de 2008 e dezembro de 2009, foram realizadas 953 angioplastias coronárias e 2.066 cateterismos cardíacos no Serviço de Hemodinâmica e Cardiologia Intervencionista do Hospital Stella Maris.

A população estudada foi composta de 128 pacientes portadores de síndrome coronária aguda ou angina estável, 66 pacientes alocados no grupo A $(61,1 \%$ do sexo masculino, média de idade de $59 \pm 8,4$ anos) e 62 pacientes no grupo B $(67,7 \%$ do sexo masculino, média de idade de $58 \pm 11,1$ anos). A média de idade dos pacientes do sexo feminino foi de $64 \pm 8,3$ anos e de $62 \pm 12,1$ anos nos grupos $\mathrm{A}$ e $\mathrm{B}$, respectivamente. Quando comparados os dois grupos em relação à idade, não foram observadas alterações estatisticamente significantes $(P=0,08)$.

Quando avaliada a presença de estresse emocional na fase pré-angioplastia, observou-se não haver diferença entre os pacientes dos dois grupos $(84,8 \%$ vs. $80,6 \%$; $P=0,69$ ); na fase pós-angioplastia, observou-se diminuição do estresse nos pacientes do grupo A e aumento nos pacientes do grupo B $(25,7 \%$ vs. 93,5\%; P $<0,00001)$.

Observa-se que os pacientes do grupo B, na primeira e na segunda fases da pesquisa, apresentaram poucas mudanças no grau de estresse, visto que em alguns quadros os sintomas do estresse emocional prevaleceram e em outros, até aumentaram (Tabela 1).

$\mathrm{Na}$ Tabela 2 observa-se que nos pacientes do grupo A, na fase pré-angioplastia, ocorreu predomínio da fase de resistência, com evolução para ausência de estresse emocional no período pós-angioplastia (74,2\%). No grupo B também se observa predomínio da fase de resistência na pré-angioplastia, mas com reduzido número de pacientes sem estresse emocional no período pós-angioplastia $(6,4 \%)$. 
Na Tabela 3 observa-se que nos pacientes dos grupos A e B ocorreu predomínio de sintomas psíquicos no período pré-angioplastia e que somente no grupo A ocorreu diminuição dos sintomas psíquicos no período pós-angioplastia. Em relação aos sintomas físicos, observa-se também redução no período pósangioplastia no grupo A.

TABELA 1

Distribuição das fases de estresse emocional entre os grupos A e B nas etapas pré e pós-angioplastia

\begin{tabular}{lccc}
\hline Variáveis & Grupo A & Grupo B & Valor de P \\
\hline Número de pacientes & 66 & 62 & \\
Alerta - pré & 2 & 2 & $>0,99$ \\
Alerta - pós & 0 & 7 & $<0,01$ \\
Resistência - pré & 37 & 35 & 0,89 \\
Resistência - pós & 13 & 35 & $<0,01$ \\
Quase exausto - pré & 9 & 6 & 0,67 \\
Quase exausto - pós & 1 & 12 & $<0,01$ \\
Exausto - pré & 8 & 7 & 0,90 \\
Exausto - pós & 3 & 4 & 0,93 \\
Sem estresse - pré & 10 & 12 & 0,69 \\
Sem estresse - pós & 49 & 4 & $<0,01$ \\
\hline
\end{tabular}

Os atendimentos psicológicos hospitalares, direcionados aos pacientes do grupo A, foram considerados eficazes por 64 pacientes (97\%).

\section{DISCUSSÃO}

O presente estudo demonstrou que a maioria dos pacientes que se submetem a angioplastia coronária apresenta estresse emocional e que o atendimento psicológico prévio na fase hospitalar se mostra benéfico na redução desse estresse. Ressalta-se, dessa forma, a importância do papel do psicólogo hospitalar na equipe de cardiologia intervencionista, atendendo os pacientes que necessitam se submeter a angioplastia coronária. O psicólogo hospitalar tem atuação limitada em relação à doença orgânica, área específica da medicina; no entanto, pode dar assistência ao paciente com relação ao significado de seus sintomas, focando sua atenção no indivíduo e não na doença. Essa é a filosofia da psicologia hospitalar, ou seja, esclarecer o paciente em relação a sua doença. ${ }^{13}$

Averiguou-se, por meio dos relatos dos pacientes participantes desta pesquisa, que desde o momento em que o paciente é informado da necessidade de se submeter a angioplastia coronária seu estado emocional se altera. Nesses relatos há queixas de insônia, aumento de sudorese, mudança de apetite, choro,

TABELA 2

Comparação das fases de estresse emocional em cada grupo nas etapas pré e pós-angioplastia

\begin{tabular}{|c|c|c|c|c|c|}
\hline & Alerta & Resistência & Quase exaustão & Exaustão & Sem estresse \\
\hline \multicolumn{6}{|l|}{ Grupo A } \\
\hline - Pré & 2 & 37 & 9 & 8 & 10 \\
\hline - Pós & 0 & 13 & 1 & 3 & 49 \\
\hline - Valor de P & 0,48 & $<0,01$ & 0,01 & 0,08 & 0,02 \\
\hline \multicolumn{6}{|l|}{ Grupo B } \\
\hline - Pré & 2 & 35 & 6 & 7 & 12 \\
\hline - Pós & 7 & 35 & 12 & 4 & 4 \\
\hline - Valor de P & 0,07 & $>0,99$ & 0,77 & 0,28 & 0,01 \\
\hline
\end{tabular}

TABELA 3

Comparação dos sintomas físicos e psíquicos em cada grupo nas etapas pré e pós-angioplastia

\begin{tabular}{lcccc} 
& Físico & Psíquico & Físico + psíquico & Sem estresse \\
\hline Grupo A & 17 & & & 10 \\
- Pré & 5 & 32 & 4 & 49 \\
- Pós & $<0,01$ & $<0,01$ & 0,25 & $<0,01$ \\
- Valor de P & 14 & & 4 & 12 \\
Grupo B & 9 & 32 & 10 & 4 \\
- Pré & 0,07 & 39 & 0,04 & 0,01 \\
- Pós & & 0,02 & \\
- Valor de P & & & & 4 \\
\hline
\end{tabular}



Invasiva. 2010;18(3):311-5.

"nervosismo" e pesadelos. Essas queixas fazem parte dos sintomas do estresse emocional. Além disso, é bastante comum a indecisão do paciente em se submeter ao tratamento, principalmente pela falta de informação relativa ao procedimento. ${ }^{15}$

No Hospital Stella Maris, entidade filantrópica com atendimento predominante aos pacientes do Sistema Único de Saúde (SUS), observam-se indivíduos com baixo nível educacional e com muitas dúvidas e fantasias a respeito da angioplastia coronária (anestesia local ou geral, extensão da incisão, desconhecimento da prótese que vai receber, etc.). A correlação entre baixo nível educacional e doença arterial coronária é relacionada a estresse psicossocial e estilo de vida não saudável. , $^{3,16,17}$

Embora o atendimento psicológico hospitalar busque situar o indivíduo diante de si mesmo, da vida e do cenário no qual está inserido, ocorrem limitações desse atendimento. Certamente, o hospital não é o local ideal para abordar aspectos psicológicos de grande profundidade, pois, em decorrência da exiguidade de tempo, muitas vezes o paciente não terá como elaborar o que foi dito. Entende-se que não é ético o psicólogo levantar questões mais profundas, sabendo que não poderá dar prosseguimento ao atendimento do paciente. ${ }^{15}$

É importante ressaltar que todos os pacientes do estudo receberam os mesmos cuidados interdisciplinares: médicos, enfermeiros e auxiliares de enfermagem do Serviço de Hemodinâmica do Hospital Stella Maris.

Face ao exposto, pode-se perceber que o papel do psicólogo hospitalar na equipe de cardiologia intervencionista é o de trabalhar as emoções do paciente, aliviando seu sofrimento psíquico, estimular a necessidade de mudanças em seus hábitos de vida, e aumentar sua expectativa quanto ao sucesso do tratamento.

\section{CONCLUSÃO}

A avaliação da eficácia do atendimento psicológico hospitalar, nos pacientes submetidos a intervenções coronárias percutâneas, permitiu demonstrar alta prevalência de estresse pré-intervenção e constatar o benefício do atendimento especializado na redução desse fenômeno.

\section{CONFLITO DE INTERESSES}

Os autores declararam inexistência de conflito de interesses relacionado a este artigo.

\section{REFERÊNCIAS}

1. Lipp MEN. Estresse emocional: a contribuição de estressores internos e externos. Rev Psiq Clín (São Paulo). 2001;28(6): 347-9.

2. Lipp MEN. Manual do Inventário de Sintomas de Stress para Adultos (ISSL). São Paulo: Casa do Psicólogo; 2002. p. 55.

3. Hornstein C. Stress, anxiety and cardiovascular disease: an interdisciplinary approach. Vertex. 2004;15 Suppl 1:21-31.

4. Alboni P, Alboni M. Psychosocial factors as predictors of atherosclerosis and cardiovascular events: contribution from animal models. G Ital Cardiol (Rome). 2006;7(11):747-53.

5. Ballone GJ. Personalidade tipo A e cardiologia. In: PsiqWeb [Internet]. [citado 2010 jul. 25]. Disponível em: www.psiqweb. med.br

6. Vale S. Psychosocial stress and cardiovascular diseases. Postgrad Med J. 2005;81(957):429-35.

7. Roest AM, Martens EJ, de Jone P, Denollet J. Anxiety and risk of incident coronary heart disease: a meta-analysis. J Am Coll Cardiol. 2010;56(1):38-46.

8. Janszky I, Ahnve S, Lundberg I, Hemmingsson T. Earlyonset depression, anxiety and risk of subsequent coronary heart disease: 37 year follow-up of 49.321 young Swedish men. J Am Coll Cardiol. 2010;56(1):31-7.

9 Hallage AG. Papel das emoções e das situações psicologicamente estressantes na gênese das arritmias cardíacas e da morte súbita. In: Lamosa BWR. Psicologia aplicada à cardiologia. São Paulo: Fundo Editorial BYK; 1992. p. 71-8.

10. Oliveira MFP, Sharosvsky LL, Ismael SMC. Aspectos emocionais no paciente coronariano. In: Oliveira MFP, Ismael SMC. Rumos da psicologia hospitalar em cardiologia. Campinas: Papirus; 1995. p.185-98.

11. Lamosa BWR. Psicologia aplicada à cardiologia: aspectos históricos. São Paulo: Fundo Editorial BYK; 1992. p. 124.

12. Silva CM, Meireles G, Abreu L, Forte A, Hayashi J. Avaliação do nível de ansiedade no pré e no pós-implante de stent coronariano com e sem sedação. Rev Soc Cardiol Estado de São Paulo. 2007;1 Supl A:3-8.

13. Simonetti A. Manual de psicologia hospitalar: o mapa da doença. São Paulo: Casa do Psicólogo; 2004. p. 201.

14. Lopes MACQ, Barros MAV, Oliveira IR, Martins HC, Paiva MS, Lima JAC, et al. Comparação do perfil epidemiológico clínico e dos resultados das intervenções coronárias percutâneas entre os gêneros masculino e feminino na população brasileira: dados do registo CENIC. Rev Bras Cardiol Invasiva. 2008;16(4):463-73.

15. Romano BW. Princípios para a prática da psicologia em hospitais. São Paulo; 1999. p. 145.

16. Wamala SP, Mittleman MA, Schenck-gustafson K, Orth-Gomér K. Potential explanations for the educational gradient in coronary heart disease: a population-based case-control study of Swedish women. Am J Public Heath. 1999;89(3):315-21.

17. Campos CAHM, Quadros AS, Welter DI, Camozzato F, Rovinski D, Sarmento-Leite R, et al. Associação entre renda, características clínicas e angiográficas de pacientes submetidos a intervenção coronária percutânea. Rev Bras Cardiol Invasiva. 2010;18(1):44-9. 